\title{
Avaliação da qualidade de vida de pacientes diabéticos tipo 2 e a prevalência de déficit sensitivo em membros inferiores
}

Hengrid Graciely Nascimento Heng
Silva

hengridgns@gmail.com

orcid.org/0000-0002-7362-0118

Universidade Estadual do Piauí (UESPI), Teresina, Piauí, Brasil

\section{Renata Miranda de Araujo Laet} Lopes

renataa miranda@hotmail.com

Universidade Estadual do Piauí (UESPI),

Teresina, Piauí, Brasil

\section{Maura Cristina Porto Feitosa} mauraportofisio@hotmail.com orcid.org/0000-0001-7095-7228 Universidade Estadual do Piauí (UESPI), Teresina, Piauí, Brasil

\section{Karen de Freitas Sousa} kfffisioterapia@gmail.com orcid.org/0000-0002-9999-7490 Universidade Estadual do Piauí (UESPI) Teresina, Piauí, Brasil

\section{Rauirys Alencar de Oliveira} raoliveira@uninovafapi.edu.br orcid.org/0000-0001-5123-004X Universidade Estadual do Piauí (UESPI) Teresina, Piauí, Brasil

\author{
RESUMO
}

OBJETIVO: Avaliar a qualidade de vida e a sensibilidade dos pés de pacientes diabéticos.

MÉTODOS: Estudo quantitativo, transversal, com 40 portadores de diabetes tipo 2. Utilizouse o questionário SF-36 e os monofilamentos de Semmes-Weinstein. Os dados foram coletados após a avaliação neurológica, tabulados e analisados por meio do software Statistica 7.0 StatSoft (C). Para comparar os grupos de indivíduos com alterações sensitivas com o grupo que não possuía alterações sensitivas foi utilizado o teste não-paramétrico U de Mann-Whitney. Foram considerados significantes os valores de $p \leq 0,05$.

RESULTADOS: Dos 40 sujeitos, $62,5 \%$ eram mulheres, com idade média de $57 \pm 9,8$ anos. 0 índice de massa corporal foi de $26,4 \pm 3,4 \mathrm{~kg} / \mathrm{m} 2$ e o tempo de diagnóstico da doença foi de $8,9 \pm 7,7$ anos. Notou-se que os aspectos emocionais (com e sem alteração sensitiva média de $52,0 \%$ e $87,5 \%$, respectivamente) e físicos (com e sem alteração sensitiva média de $47,7 \%$ e $71,9 \%$, respectivamente) foram os que apresentaram maior diferença, embora, não estatisticamente significante.
\end{abstract}

CONCLUSÕES: A qualidade de vida dos pacientes sem alteração sensitiva foi melhor em relação à maioria dos domínios. Não houve alteração na qualidade de vida comparada ao comprometimento sensorial estatisticamente significante, mas na maioria dos domínios do SF-36 os pacientes sem alteração sensitiva apresentaram melhor qualidade de vida.

PALAVRAS-CHAVE: Qualidade de vida. Diabetes Mellitus. Pé diabético. 


\section{INTRODUÇÃO}

A diabetes mellitus (DM) é considerada uma das principais doenças crônicas da atualidade. Em virtude de sua grande incidência no cenário mundial e de complicações relacionadas a essa doença, é uma anormalidade endócrinometabólica complexa, multifatorial e de presença global, que pode levar a complicações micro e macrovasculares (SALOMÉ; BLANES; FERREIRA, 2011; ARAÚJO et al., 2013).

A prevalência de DM nos países da América Central e do Sul foi estimada em 26,4 milhões de pessoas e projetada para 40 milhões, em 2030. Nos países em desenvolvimento este aumento ocorrerá em todas as faixas etárias, sendo que no grupo de 45 a 64 anos, a prevalência será triplicada (MINISTÉRIO DA SAÚDE, 2013). No Brasil, há cerca de 7,6 milhões de diabéticos, correspondentes a $6 \%$ da população nacional (SHAW; SICREE; ZIMMET, 2010). Dados publicados pela Organização Mundial da Saúde confirmam que a prevalência da DM permanece em contínuo aumento, passando de 4,7\% em 1980 a 8,5\% em 2014 e tem sido a causa de mais de 1,5 milhões disfunções em 2012 (WORLD HEALTH ORGANIZATION, 2016).

Tanto a doença quanto seu tratamento exigem dos pacientes alterações importantes no cotidiano, além do impacto na vida de suas famílias, amigos e comunidade. Por se tratar de uma doença progressiva, os indivíduos acometidos tendem a deteriorar seu estado de saúde com o passar do tempo, quando começam a aparecer as complicações derivadas de um mau controle glicêmico, o que afeta diretamente a qualidade de vida (QV) e interfere na expectativa desta (FARIA et al., 2013; SOLLI; STAVEM; KRISTIANSEN, 2010).

A diabetes mellitus tipo 2 (DM2) é o tipo mais comum, em torno de $90 \%$ a $95 \%$, e cuja prevalência está aumentando de forma exponencial, adquirindo características epidêmicas em vários países. Os fatores mais importantes que afetam a QV desses pacientes são idade, sedentarismo e obesidade. Depressão ou presença de complicações derivadas da diabetes, além de doenças concomitantes, têm um forte impacto sobre a QV desses pacientes (AMERICAN DIABETES ASSOCIATION, 2014; ALVES et al. 2012).

Entre as diversas comorbidades clínicas do DM, podem ser destacadas as microvasculares, em especial a neuropatia diabética periférica (NDP), considerada uma das principais complicações da diabetes e que afeta aproximadamente $50 \%$ dos pacientes com 20 anos de diabetes, tornando esta complicação mais comum e particularmente sintomática entre adultos mais velhos (NAJAFI; CREWS; WROBEL, 2013). A disfunção neurológica é iniciada nas porções mais distais do sistema nervoso periférico (nos pés, geralmente) e se estende às proximidades de ambas as extremidades inferiores. O comprometimento dos nervos sensitivos manifesta-se por distúrbios da sensibilidade nas extremidades, podendo chegar à anestesia (BARAZ et al., 2014).

A neuropatia sensorial periférica é um dos mais fortes fatores individuais associados ao desenvolvimento de úlceras de pé e amputações. Estas complicações afetam a QV dos pacientes, impossibilitando-os, muitas vezes, de exercer suas atividades normais, além do aumento dos custos de cuidados de saúde e internações repetidas. Por esse motivo, o pé diabético é considerado uma das mais devastadoras complicações crônicas da doença. A úlcera no pé acomete 
$15 \%$ dos indivíduos diabéticos no decorrer de sua doença e mais de $80 \%$ das amputações são precedidas de úlcera (BARAZ et al., 2014; ALMEIDA et al., 2013). Diante desses motivos, mostra-se importante realizar regularmente o exame neurológico dos pés para avaliar a sensibilidade, prevenir futuros riscos de ulceração e amputação e indicar a melhor conduta terapêutica.

O objetivo deste estudo é avaliar a qualidade de vida e a sensibilidade dos pés de pacientes diabéticos.

\section{MÉTODOS}

Trata-se de um estudo com abordagem quantitativa de perfil epidemiológico descritivo e período de segmento transversal, desenvolvido na Associação de Diabéticos do Piauí (ADIP), em Teresina, Piauí. Todos os participantes foram esclarecidos em relação aos métodos e finalidades da pesquisa, e em concordância com os mesmos assinaram um Termo de Consentimento Livre e Esclarecido (TCLE). Este projeto foi aprovado pelo Comitê de Ética em Pesquisa da Faculdade de Ciências Médicas da Universidade Estadual do Piauí (CAAE no 39600314.2.0000.5209).

Foi obtida uma amostra não-probabilística intencional de um grupo de 40 pacientes diabéticos tipo 2, maiores de 18 anos, com cognição preservada, sem úlcera nos pés e cadastrados na ADIP. Foram selecionados aleatoriamente durante as reuniões educativas mensais promovidas pela ADIP, no período de janeiro a agosto de 2015.

Os pacientes foram submetidos a um teste neurológico dos pés para avaliação dos níveis de sensibilidade dos mesmos. O teste foi realizado por uma pessoa treinada. Foi utilizado um conjunto de seis monofilamentos de Semmes-Weinstein (estesiometria) com a finalidade de avaliar e quantificar o limiar de percepção do tato e sensação de pressão profunda do pé. A percepção de cada monofilamento está associada com um gradiente de alterações sensitivas. A estesiometria é um método eficaz para detectar pés diabéticos em risco de ulceração e tem sido recomendado pela Sociedade Brasileira de Diabetes para avaliar se o pé de pacientes diabéticos está em risco de ulceração e amputação.

A sequência de pontos testados foi selecionada aleatoriamente. Iniciou-se com o monofilamento mais fino $0,05 \mathrm{~g}$ (verde), na ausência de resposta o de $0,2 \mathrm{~g}$ (azul), e assim sucessivamente, perpendicularmente à pele, com força suficiente para causar seu encurvamento por um segundo. Após definir o resultado de cada área do pé, constatou-se a presença ou não de alteração da sensibilidade e depois foi comparada com a QV do paciente. Após definir o resultado de cada área do pé, constatou-se a presença ou não de alteração da sensibilidade e depois foi comparada com a QV do paciente, comparando os indivíduos com e sem alteração.

Para avaliar a QV, foi utilizado o questionário Short Form-36 Health Survey (SF36). Este instrumento de mensuração da QV é validado no Brasil (CAMPOLINA et al., 2011). O SF-36 é um instrumento genérico, multidimensional, formado por 36 questões, que abrangem 8 domínios. A avaliação dos resultados foi feita mediante a atribuição de escores para cada questão, os quais foram transformados numa escala de 0 a 100, onde 0 correspondeu a um pior estado de saúde e 100, ao melhor. Cada dimensão foi analisada separadamente. 
Os dados foram coletados após a avaliação neurológica, tabulados e analisados por meio do software Statistica 7.0 StatSoft (C). Para comparar os grupos de indivíduos com alterações sensitivas com o grupo que não possuía alterações sensitivas foi utilizado o teste não-paramétrico $U$ de Mann-Whitney. Foram considerados significantes os valores de $p \leq 0,05$.

\section{RESULTADOS}

Dos 40 pacientes com DM2, a idade média foi de $57 \pm 9,8$ anos, sendo que a maior parte dos entrevistados tinham entre 50 e 60 anos (Figura 1). Houve predomínio do sexo feminino $(62,5 \%)$.

Figura 1 - Histograma da idade

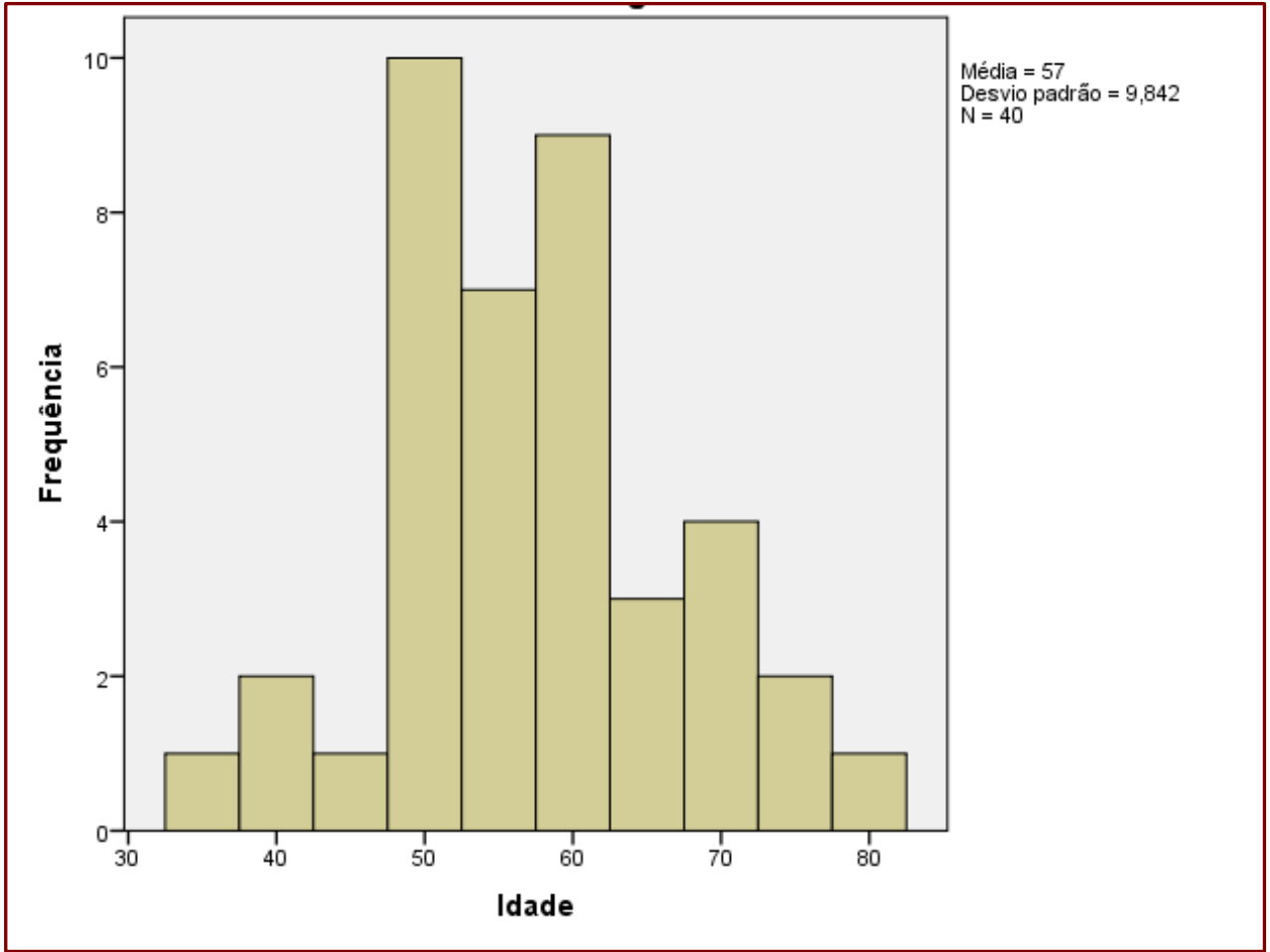

Fonte: Autoria própria (2015).

Em relação ao índice de massa corporal (IMC) e ao tempo de diagnóstico da

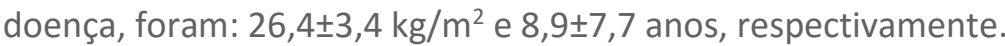

Tabela 1 - Medidas de tendência central e de dispersão para o tempo de diagnóstico de diabetes e índice de massa corpórea (IMC)

\begin{tabular}{|ccccc}
\multicolumn{1}{c}{ Variáveis } & Mínimo & Máximo & Média & Desvio padrão \\
\hline Quanto tempo tem diabetes (anos) & 0,5 & 24,0 & 8,9 & 7,7 \\
IMC $\left(\mathrm{kg} / \mathrm{m}^{2}\right)$ & 21,1 & 33,6 & 26,4 & 3,4
\end{tabular}

Fonte: Autoria própria (2015).

Observa-se na Tabela 2 que os aspectos emocionais (com alteração sensitiva: média de 52,0\%; sem alteração sensitiva: média de 87,5\%) e físicos (com alteração 
sensitiva: média de $47,7 \%$; sem alteração sensitiva: média de $71,9 \%)$ são os que aparentam maior diferença, embora, não estatisticamente significante ao adotar o nível mínimo de significância de 5\% (p-valor<0,05).

Tabela 2 - Teste U de Mann-Whitney comparando a variância entre o grupo com alteração sensitiva e o grupo sem alteração sensitiva

\begin{tabular}{|c|c|c|c|c|c|c|}
\hline Domínio & $\begin{array}{l}\text { Alteração } \\
\text { sensitiva }\end{array}$ & $\mathbf{N}$ & Média & $\begin{array}{l}\text { Desvio } \\
\text { padrão }\end{array}$ & $\begin{array}{c}\text { Erro padrão } \\
\text { da média }\end{array}$ & $\begin{array}{c}\text { Significância } \\
\text { (Bicaudal) }\end{array}$ \\
\hline \multirow{2}{*}{$\begin{array}{l}\text { Capacidade } \\
\text { funcional }\end{array}$} & Sim & 32 & 74,53 & 14,99 & 2,65 & \multirow{2}{*}{0,855} \\
\hline & Não & 8 & 73,12 & 21,53 & 7,61 & \\
\hline \multirow{2}{*}{$\begin{array}{l}\text { Aspectos } \\
\text { físicos }\end{array}$} & Sim & 32 & 47,65 & 39,83 & 7,04 & \multirow{2}{*}{0,109} \\
\hline & Não & 8 & 71,87 & 33,90 & 11,98 & \\
\hline \multirow{2}{*}{ Dor } & Sim & 32 & 65,37 & 28,68 & 5,07 & \multirow{2}{*}{0,908} \\
\hline & Não & 8 & 63,25 & 32,16 & 11,37 & \\
\hline \multirow{2}{*}{$\begin{array}{l}\text { Estado geral } \\
\text { de saúde }\end{array}$} & Sim & 32 & 60,81 & 22,43 & 3,96 & \multirow{2}{*}{0,630} \\
\hline & Não & 8 & 57,00 & 20,17 & 7,13 & \\
\hline \multirow{2}{*}{ Vitalidade } & Sim & 32 & 60,31 & 23,17 & 4,09 & \multirow{2}{*}{0,309} \\
\hline & Não & 8 & 71,25 & 9,91 & 3,50 & \\
\hline \multirow{2}{*}{$\begin{array}{l}\text { Aspectos } \\
\text { sociais }\end{array}$} & Sim & 32 & 74,84 & 24,87 & 4,39 & \multirow{2}{*}{0,249} \\
\hline & Não & 8 & 86,12 & 16,76 & 5,92 & \\
\hline \multirow{2}{*}{$\begin{array}{l}\text { Aspectos } \\
\text { emocionais }\end{array}$} & Sim & 32 & 52,03 & 47,11 & 8,32 & \multirow{2}{*}{0,095} \\
\hline & Não & 8 & 87,50 & 35,35 & 12,50 & \\
\hline \multirow{2}{*}{ Saúde mental } & Sim & 32 & 68,00 & 26,33 & 4,65 & \multirow{2}{*}{0,377} \\
\hline & Não & 8 & 82,50 & 6,02 & 2,12 & \\
\hline
\end{tabular}

Fonte: Autoria própria (2015).

De acordo com a Figura 2, verificou-se que quanto mais próximo do contorno marginal do eneágono, melhor a QV, com predominância dos pacientes sem alteração sensitiva em relação aos domínios: aspectos físicos (70\%); vitalidade (70\%); aspectos sociais (90\%); aspectos emocionais (90\%) e saúde mental $(80 \%)$, com exceção de capacidade funcional (70\%), dor (60\%) e estado geral de saúde (60\%), onde a QV dos pacientes com alteração sensitiva foi equivalente à dos indivíduos sem alteração sensitiva. 
Figura 2 - SF-36 avaliação da qualidade de vida pelos diversos domínios

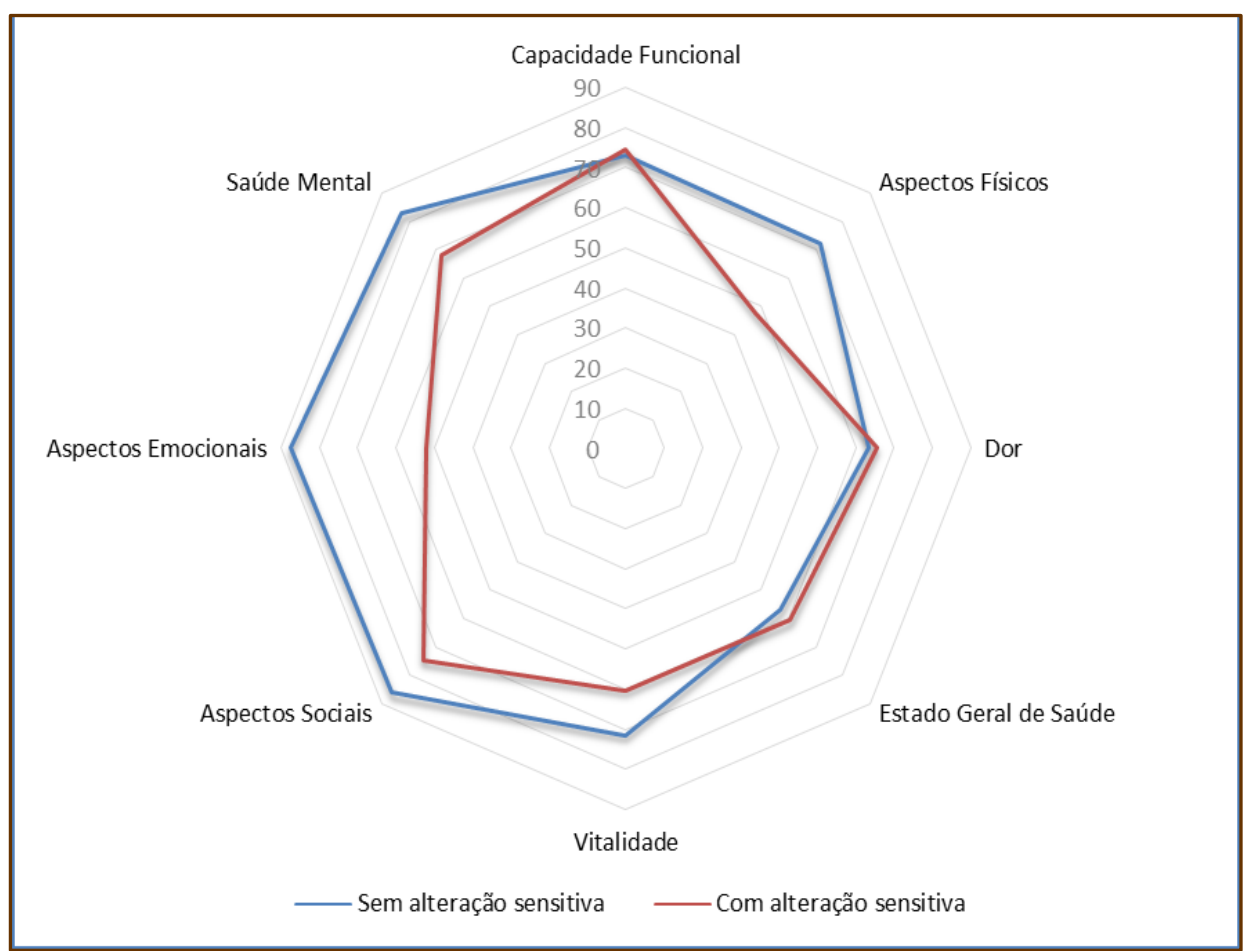

Fonte: Autoria própria (2015).

\section{DISCUSSÃO}

Segundo Araujo et al. (2013), a diabetes é particularmente prevalente entre os idosos, principalmente, as faixas etárias mais elevadas, sem distinção de raça ou condições socioeconômicas. E, em relação ao sexo, verificou-se que houve prevalência da população feminina. No estudo realizado por Alves et al. (2012), a média de idade dos participantes com DM2 foi acima de 60 anos, na sua maioria também do sexo feminino e o tempo médio de DM foi acima de 15 anos.

Em estudo realizado com o objetivo de estimar a prevalência do déficit sensitivo de membros inferiores (MMII) em indivíduos com DM2 e identificar fatores associados ao diagnóstico positivo do déficit através do escore de sintomas neuropáticos e testes de sensibilidade, foi verificado que a maioria da amostra estava acima do peso ideal e o tempo diagnostico médio da doença foi de 10,9 anos (BRINATI et al., 2017). Segundo estudo com objetivo de correlacionar o impacto da DM2 com a obesidade sobre a QV, teve como resultado que as duas condições combinadas causam uma redução nas pontuações de QV. Para avaliar a QV que foi utilizado o SF-36, ratificando os dados do presente estudo (HAJIAN; HEIDARI; HAJIAN, 2016).

Apesar de não haver alteração na QV relacionada ao comprometimento sensorial estatisticamente significante, foi perceptível na Tabela 2 e na Figura 2 que dois indicadores (aspectos emocionais e aspectos físicos) apresentaram maiores diferenças - os aspectos emocionais com alteração sensitiva média de $52,0 \%$; sem alteração sensitiva média de $87,5 \%$ ) e físicos com alteração sensitiva 
média de 47,7\%; sem alteração sensitiva média de $71,9 \%$. Esses resultados mostram que os diabéticos tendem a ter alterações na saúde física ou algum problema emocional (como se sentir deprimido ou ansioso) que interfere negativamente no seu trabalho ou em outra atividade regular diária (ALVES et al., 2012), que caracteriza depressão ou presença de complicações derivadas do DM, além de doenças concomitantes, como um forte impacto sobre a QV desses pacientes.

Ao avaliar o estado de sintomas depressivos e a QV de 210 pacientes com DM2 internados num hospital da China, foi possível observar que uma parcela dos pacientes do sexo feminino apresentava uma QV significativamente pior em decorrência a fatores psicológicos (WANG et al., 2017). As alterações sensitivas, também estão relacionadas à baixa da QV. Estudos relacionando limiar de dor em pacientes diabéticos, tem como resultado que esses pacientes estão mais susceptíveis a crises dolorosas.

Segundo Tanik et al. (2016), para pacientes diabéticos a cronificação da dor neuropática podem levar à deterioração de fatores que alteram a QV. A neuropatia periférica diabética dolorosa é uma das formas mais comuns de dor neuropática e a sua incidência aumenta à medida que a obesidade e as epidemias de diabetes continuam a crescer. A neuropatia é causada por fatores metabólicos, bem como pelo dano à microvasculatura que fornece fibras nervosas (HOLMES, 2016).

Diferente deste estudo, na avaliação da QV dos participantes, observou-se que apenas o domínio capacidade funcional do SF-36 apresentou valor mediano abaixo de 50, o que corresponde à metade do escore máximo para cada componente, mostrando assim, maior comprometimento. Os domínios aspectos sociais, aspectos emocionais e aspectos físicos foram os que apresentaram melhor avaliação. Entretanto, é comum o comprometimento no domínio capacidade funcional entre esses pacientes, até porque se trata de uma doença progressiva, os indivíduos acometidos tendem a deteriorar seu estado de saúde com o passar do tempo, quando começam a aparecer às complicações derivadas do mau controle glicêmico (FARIA et al., 2013).

No estudo realizado por Leal et al. (2014) com 100 diabéticos, 59\% eram do sexo feminino, a idade variou de 43 anos (média=64,1 anos) e o tempo médio do diagnóstico do DM foi 8,6 anos. Na avaliação da QV obtiveram-se os escores médios padronizados para cada dimensão do SF-36, onde os valores variaram de 34,8 a 72,0 caracterizando o maior e o menor impacto da diabetes na QV. Em três dimensões constatou-se um valor de escore mediano abaixo de 50 (aspectos físicos, aspectos emocionais e estado geral de saúde), coincidindo com os componentes de maior comprometimento deste estudo, com exceção do domínio estado geral de saúde (LEAL et al., 2014).

No estudo realizado por Baraz et al. (2014), também com pacientes DM2 sem úlcera nos pés, com uma condição clínica estável, sem nenhum problema psicológico, com nenhum calo ou quaisquer outras complicações em pés e sendo capaz de comunicar-se verbalmente, foram utilizados os monofilamentos de Semmes-Weinstein. O teste neurológico foi realizado em ambos os pés dos pacientes em 3, 4, 8, e 10 pontos dos pés. Em cada ponto, o teste foi repetido por três vezes. Se o paciente respondeu incorretamente duas ou mais vezes em que ponto sentiu o monofilamento, ele foi gravado como um sintoma positivo de neuropatia. Verificou-se que 93 pacientes eram saudáveis e sem neuropatia e 57 
indivíduos apresentaram neuropatia, ou seja, a menor porção dos indivíduos apresentou déficit sensitivo nos pés, talvez porque recebem tratamento adequado e precoce em clínicas diabéticas.

A alteração de sensibilidade nos pés esteve presente na maioria dos participantes $(80 \%)$ deste estudo, confirmando que a neuropatia diabética periférica é uma das complicações mais frequentes da DM. Não obstante, essa complicação não interferiu significativamente na QV deles segundo análise estatística, provavelmente porque o grupo controle foi pequeno ou até mesmo porque os indivíduos são informados sobre a doença e suas complicações e tomam o devido cuidado; corroborando com o estudo realizado num ambulatório de geriatria com o objetivo de avaliar a QV de 62 pacientes diabéticos. A pesquisa teve como resultado que não houve diferença estatística significativa na QV de pacientes diabéticos no quesito funcionamento do sensório (ESTEVES et al., 2017). Contudo, segundo Macioch et al. (2017), a QV de pacientes com DM2 é afetada de forma significativa se o doente vier a ter úlcera do pé diabético (UPD), que é uma complicação comum da diabetes e um importante fator de mortalidade entre os pacientes com diabetes, o que não é o caso desse estudo, já que a amostra analisada não possui indivíduos com UPD.

Segundo Yan et al. (2014), um dos fatores pré-hospitalares que podem influenciar no atraso prolongado da UPD e melhora da QV de pacientes diabéticos, são os programas de intervenção que atendem às comunidades em risco para incentivar a avaliação da equipe multidisciplinar, o conhecimento sobre os riscos da doença e o autocuidado dos pacientes com diabetes. Assim, este modelo de atenção ao diabético pode fortalecer as relações grupais existentes ou promover novas afiliações que formam a base para futuras coalizões de ação. Os participantes trabalharam tanto dentro como entre grupos para trocar informações, histórias de sucesso e desafios e estratégias específicas de melhoria da saúde e de QV (ZIEGAHN et al., 2014).

O uso exclusivo dos monofilamentos ou em combinação com outros testes de reflexo para método de triagem da neuropatia é um método fácil e acessível; e pela detecção precoce, pode prevenir complicações que incluem úlceras de perna e amputação em pacientes com diabetes.

As limitações deste estudo concentraram-se na dificuldade de abordar aos pacientes que queriam prestar atenção na palestra dada no momento da reunião, além da dificuldade de compreensão imediata em relação às categorias de resposta de determinados itens do instrumento SF-36 por parte de alguns participantes, sendo necessário reler as perguntas e explicar para eles de maneira mais clara.

Este estudo mostrou que não há alteração na QV comparada ao comprometimento sensorial estatisticamente significante, provavelmente devido ao grupo controle pequeno e às condições de acesso à saúde. Recomenda-se que novos estudos sejam feitos com uma amostra maior para que fiquem mais evidentes as influências da alteração de sensibilidade sobre os aspectos da QV. 


\title{
Quality of life evaluation in type 2 diabetic patients and sensitive deficit prevalence in lower extremity
}

\begin{abstract}
OBJECTIVE: To evaluate and compare a quality of life and sensitivity of the feet of diabetic patients.

METHODS: Quantitative cross-sectional study was performed with 40 patients with type 2 diabetes. The SF-36 questionnaire and Semmes-Weinstein monofilaments were used. The data were worked by number and percentage and the statistical test used was the MannWhitney $\mathrm{U}$, which evaluated the degree of interlacing of the data of the two groups after the ordering, with a confidence interval of $95 \%$ and significance at $p<0,05$.

RESULTS: Of the 40 subjects, $62.5 \%$ were women, with a mean age of $57 \pm 9.8$ years. The body mass index was $26.4 \pm 3.4 \mathrm{~kg} / \mathrm{m} 2$ and the disease diagnosis time was $8.9 \pm 7.7$ years. It was observed that the emotional aspects (with and without mean sensitivity change of $52.0 \%$ and $87.5 \%$ respectively) and physical (with and without mean sensory change of $47.7 \%$ and $71.9 \%$, respectively) were those that Presented a greater difference, although not statistically significant.

CONCLUSIONS: The quality of life of patients unchanged sensitive were better compared to most domains: physical, social and emotional aspects, vitality and mental health with the exception of functional capacity, pain and general health where the quality of life of the two groups was equivalent. There is no change in quality of life compared to sensory impairment statistically significant, but it was found that in most domains of the SF-36 patients without sensory change had better quality of life.
\end{abstract}

KEYWORDS: Quality of life. Diabetes mellitus. Diabetic foot. 


\section{REFERÊNCIAS}

ALMEIDA, S. A. et al. Avaliação da qualidade de vida em pacientes com diabetes mellitus e pé ulcerado. Revista Brasileira de Cirurgia Plástica, v. 28, n. 1, p. 142146, 2013. Disponível em:

$<$ https://www.researchgate.net/profile/Geraldo Magela Salome2/publication/2 62519362 Assessment of the quality of life of patients with diabetes mellit us and foot ulcers/links/54b67f1e0cf24eb34f6d2572.pdf>. Acesso em: 30 jun. 2017. Crossef

ALVES, T. O. S. et al. Qualidade de vida relacionada à saúde de pessoas com diabetes mellitus. Revista Mineira de Enfermagem, v. 17, n. 1, p. 136-148, 2012. Disponível em: <http://www.reme.org.br/artigo/detalhes/585>. Acesso em: 30 jun. 2017.

AMERICAN DIABETES ASSOCIATION. Diagnosis and classification of diabetes mellitus. Diabetes Care, v. 37, p. S81-S90, 2014. Disponível em: $<$ http://care.diabetesjournals.org/content/37/Supplement 1/S81.short>. Acesso em: 29 jun. 2017. Crossef

ARAÚJO, K. O. et al. Avaliação da qualidade de vida de portadores de diabetes mellitus tipo 2. Revista de Enfermagem UFPE, v. 7, n. 9, p. 5583-5589, 2013. Disponível em: $<$ https://periodicos.ufpe.br/revistas/revistaenfermagem/article/view/13677>. Acesso em: 30 jun. 2017.

BARAZ, S. et al. Comparison of the accuracy of monofilament testing at various points of feet in peripheral diabetic neuropathy screening. Journal of Diabetes \& Metabolic Disorders, v. 13, n. 1, p. 1-7, 2014. Disponível em: $<$ https://jdmdonline.biomedcentral.com/articles/10.1186/2251-6581-13-19>. Acesso em: 30 jun. 2017. Crossef

BRINATI, L. M. et al. Prevalência e fatores associados à neuropatia periférica em indivíduos com diabetes mellitus. Revista de Pesquisa: Cuidado é Fundamental Online, v. 9, n. 2, p. 347-355, 2017. Disponível em:

<http://www.seer.unirio.br/index.php/cuidadofundamental/article/view/4476>. Acesso em: 30 jun. 2017.

CAMPOLINA, A. G. et al. Validação da versão brasileira do questionário genérico de qualidade de vida short-form 6 dimensions (SF-6D Brasil). Ciência \& Saúde Coletiva, v. 16, n. 7, p. 3103-3110, 2011. Disponível em:

$<$ http://www.scielo.br/pdf/csc/v16n7/10.pdf>. Acesso em: 22 jun. 2017. Crossef 
ESTEVES, M. et al. Qualidade de vida de idosos hipertensos e diabéticos em um serviço ambulatorial. Medicina, v. 50, n. 1, p. 18-28, 2017. Disponível em: $<$ http://revista.fmrp.usp.br/2017/vol50n1/AO3-Qualidade-de-vida-de-idososhipertensos-e-diabeticos.pdf $>$. Acesso em: 29 jun. 2017.

FARIA, H. T. G. et al. Qualidade de vida de pacientes com diabetes mellitus antes e após participação em programa educativo. Revista da Escola de Enfermagem da USP, v. 47, n. 2, p. 348-354, 2013. Disponível em:

<http://www.redalyc.org/html/3610/361033325011/>. Acesso em: 30 jun. 2017. crossef

HAJIAN, T. K.; HEIDARI, B.; HAJIAN, T. A. Solitary and combined negative influences of diabetes, obesity and hypertension on health-related quality of life of elderly individuals: a population-based cross-sectional study. Diabetes \& Metabolic Syndrome: Clinical Research \& Reviews, v. 10, n. 2, p. S37-S42, 2016. Disponível em: <http://www.sciencedirect.com/science/article/pii/S1871402115300746>. Acesso em: 29 jun. 2017. Crossef

HOLMES, D. The pain drain. Nature, v. 535, p. S2, 2016. Disponível em: $<$ https://www.nature.com/nature/journal/v535/n7611 supp/full/535S2a.html $>$. Acesso em: 29 jun. 2017. Crossef

LEAL, L. B. et al. Qualidade de vida relacionada à saúde de pessoas com diabetes mellitus tipo 2. Northeast Network Nursing Journal, v. 15, n. 4, p. 676-682, 2014. Disponível em:

$<$ http://www.periodicos.ufc.br/index.php/rene/article/view/1101>. Acesso em: 30 jun. 2017.

$\mathrm{MACIOCH}, \mathrm{T}$. et al. Health related quality of life in patients with diabetic foot ulceration -translation and Polish adaptation of Diabetic Foot Ulcer Scale short form. Health and Quality of Life Outcomes, v. 15, n. 1, p. 15, 2017. Disponível em: <https://hqlo.biomedcentral.com/articles/10.1186/s12955-017-0587-y>. Acesso em: 29 jun. 2017. Crossef

MINISTÉRIO DA SAÚDE. Secretaria de Atenção à Saúde. Departamento de Atenção Básica. Estratégias para o cuidado da pessoa com doença crônica: diabetes mellitus. Brasília: Ministério da Saúde, 2013. Disponível em: $<$ http://bvsms.saude.gov.br/bvs/publicacoes/estrategias cuidado pessoa diabet es mellitus cab36.pdf>. Acesso em: 15 jun. 2017. 
NAJAFI, B.; CREWS, R. T.; WROBEL, J. S. A novel plantar stimulation technology for improving protective sensation and postural control in patients with diabetic peripheral neuropathy: a double-blinded, randomized study. Gerontology, v. 59, n. 5, p. 473-480, 2013. Disponível em: $<$ https://www.karger.com/Article/PDF/352072>. Acesso em: 30 jun. 2017. crossef

SALOMÉ, G. M.; BLANES, L.; FERREIRA, L. M. Avaliação de sintomas depressivos em pessoas com diabetes mellitus e pé ulcerado. Revista do Colégio Brasileiro de Cirurgiões, v. 38, n. 5, p. 327-333, 2011. Disponível em:

<http://vml029.epm.br/handle/11600/6661>. Acesso em: 30 jun. 2017. Crossef

SHAW, J. E.; SICREE, R. A.; ZIMMET, P. Z. Global estimates of the prevalence of diabetes for 2010 and 2030. Diabetes Research and Clinical Practice, v. 87, n. 1, p. 4-14, 2010. Disponível em:

<http://www.sciencedirect.com/science/article/pii/S016882270900432X>. Acesso em: 30 jun. 2017. Crossef

SOLLI, O.; STAVEM, K.; KRISTIANSEN, I. S. Health-related quality of life in diabetes: The associations of complications with EQ-5D scores. Health and Quality of Life Outcomes, v. 8, n. 1, p. 18, 2010. Disponível em: <https://hqlo.biomedcentral.com/articles/10.1186/1477-7525-8-18>. Acesso em: 30 jun. 2017. Crossef

TANIK, N. et al. Pain, depression and sleep disorders in patients with diabetic and nondiabetic carpal tunnel syndrome: a vicious cycle. Arquivos de Neuro-

Psiquiatria, v. 74, n. 3, p. 207-211, 2016. Disponível em:

$<$ http://www.scielo.br/scielo.php?script=sci arttext\&nrm=iso\&lng=pt\&tlng=pt\&p id=S0004-282X2016000300006 >. Acesso em: 29 jun. 2017. Crossef

WANG, X. et al. Investigating factors associated with depressive symptoms of chronic kidney diseases in china with type 2 diabetes. Journal of Diabetes Research, v. 2017, p. 1-7, 2017. Disponível em: <https://www.hindawi.com/journals/jdr/2017/1769897/abs/>. Acesso em: 29 jun. 2017. Crossef

WORLD HEALTH ORGANIZATION (WHO). Global report on diabetes. Geneva: World Health Organization, 2016. Disponível em: <http://apps.who.int/iris/bitstream/10665/204871/1/9789241565257 eng.pdf>. Acesso em: 30 jun. 2017. 
YAN, J. et al. Pre-hospital delay in patients with diabetic foot problems:

influencing factors and subsequent quality of care. Diabetic Medicine, v. 31, n. 5, p. 624-629, 2014. Disponível em:

$<$ http://onlinelibrary. wiley.com/doi/10.1111/dme.12388/abstract;jsessionid=432 4BF2BD861A5C08660D3AA6A4C8A7E.f03t02? systemMessage=Wiley+Online+Libr ary+\%27Journal+Subscribe+\%2F+Renew $\% 27+$ page+will+be+down+on+Wednesda $y+05$ th+July+starting+at+08.00+EDT+\%2F+13.00+BST+\%2F+17.30+IST+for+up+to +75+minutes+due+to+essential+maintenance. $>$. Acesso em: 29 jun. 2017. crossef

ZIEGAHN, L. et al. Strategies to prevent and reduce diabetes and obesity in Sacramento, California: the African American leadership coalition and university of California, Davis. Preventing Chronic Disease, v. 10, 2013. Disponível em: <https://www.ncbi.nlm.nih.gov/pmc/articles/PMC3830925/?tool=pubmed.>. Acesso em: 29 jun. 2017.

\section{(c) (i)}

